\title{
Resistência a carbapenêmicos por Acinetobacter spp. reportados ao GLASS de acordo com o nível de renda dos países
}

\author{
Resistance to carbapenems by Acinetobacter spp. reported to GLASS according to the income level \\ of the countries
}

Resistencia a los carbapenémicos por Acinetobacter spp. reportado a GLASS según el nivel de ingresos de los países

\section{Resumo}

Este trabalho tem por objetivo avaliar a resistência a carbapenêmicos da Acinetobacter spp. reportada ao GLASS (Global Antimicrobial Resistance and Use Surveillance System), estratificando esses dados por faixa de renda per capita. Foi conduzido um estudo observacional retrospectivo sobre a sensibilidade a carbapenêmicos da Acinetobacter spp., reportada ao GLASS, fora incluído todos os dados disponíveis no momento da coleta, fevereiro de 2021, estratificando esses achados pelo nível de renda de cada país e a quantidade de testes de sensibilidade a carbapenêmicos realizados. Os países de maior renda tiveram a menor não-suscetibilidade relatada, contudo dentro deles existem exemplos que apresentam uma alta incidência de não-suscetibilidade. Para todos os quatro grupos de renda existiu uma alta variância do desvio padrão para a média dos testes de sensibilidade a carbapenêmicos, assim revelando pontos de inconsistência. Os dados trabalhados neste artigo mostram um nível preocupante de resistência a carbapenêmicos observados no GLASS. Dentro mesmo dos países ricos há aqueles que possuem níveis alarmantes de não-susceptibilidade e, para os países mais pobres, destaca-se uma escassez de dados mais completos, pois naqueles com os níveis mais preocupantes de não-susceptibilidade já são encontradas cepas de Acinetobacter spp. com fenótipo de multirresistência.

Palavras-chave: Testes de sensibilidade microbiana; Uso racional de medicamentos; Monitoramento epidemiológico; Resistência bacteriana a antibióticos.

\footnotetext{
Abstract

This work aims to evaluate the resistance to carbapenems of Acinetobacter spp. reported to the GLASS (Global Antimicrobial Resistance and Use Surveillance System), stratifying these data by per capita income bracket. A retrospective observational study was conducted on carbapenem sensitivity of Acinetobacter spp., reported to GLASS,
} 
including all data available at the time of collection, february 2021, stratifying these findings by each country's income level and number of tests of sensitivity to carbapenemics performed. Higher-income countries had the lowest reported non-susceptibility, yet within them there are examples that have a high incidence of non-susceptibility. For all four income groups there was a high standard deviation variance for the mean of the carbapenem sensitivity tests, thus revealing points of inconsistency. The data worked in this article show a worrying level of resistance to carbapenems observed in GLASS. Even within rich countries, there are those that have alarming levels of nonsusceptibility, and for the poorest countries, there is a lack of more complete data, because in those with the most worrying levels of non-susceptibility, strains of Acinetobacter spp. are already found with multidrug resistance phenotype.

Keywords: Microbial sensitivity tests; Drug utilization; Epidemiological monitoring; Drug resistance bacterial.

\section{Resumen}

Este trabajo tiene como objetivo evaluar la resistencia a los carbapenémicos de Acinetobacter spp. informaron al GLASS (Global Antimicrobial Resistance and Use Surveillance System), estratificando estos datos por tramo de ingreso per cápita. Se realizó un estudio observacional retrospectivo sobre la sensibilidad a carbapenémicos de Acinetobacter spp., reportado a GLASS, incluyendo todos los datos disponibles al momento de la recolección, febrero de 2021, estratificando estos hallazgos por nivel de ingresos de cada país y número de pruebas de sensibilidad a carbapenémicos realizadas. Los países de ingresos más altos registraron la no susceptibilidad más baja, pero dentro de ellos hay ejemplos que tienen una alta incidencia de no susceptibilidad. Para los cuatro grupos de ingresos hubo una alta variación de desviación estándar para la media de las pruebas de sensibilidad a los carbapenémicos, lo que reveló puntos de inconsistencia. Los datos trabajados en este artículo muestran un nivel preocupante de resistencia a los carbapenémicos observado en GLASS. Incluso dentro de los países ricos, hay aquellos que tienen niveles alarmantes de no susceptibilidad y, para los países más pobres, hay una falta de datos más completos, porque en aquellos con los niveles más preocupantes de no susceptibilidad, las cepas de Acinetobacter spp. ya están encontradas con fenotipo de resistencia a múltiples fármacos.

Palabras clave: Pruebas de sensibilidad microbiana; Utilización de medicamentos; Monitoreo epidemiológico; Farmacorresistencia bacteriana.

\section{Introdução}

Carbapenêmicos são antimicrobianos beta-lactâmicos utilizados em infecções graves causadas por bactérias multirresistentes, sendo algumas vezes a última linha de tratamento disponível. Esses beta-lactâmicos apresentam mecanismo de ação semelhante ao das penicilinas, inibindo a síntese da parede celular, eles possuem ampliado espectro em comparação a elas, porém mesmo para eles a resistência bacteriana tem se tornado um problema crescente, principalmente para as bactérias do gênero Acinetobacter spp. (Hawkey \& Livermore, 2012). Por esse motivo o fenótipo de resistência aos carbapenêmicos dáse principalmente pelo desenvolvimento de enzimas de clivagem com espectro de ação ampliado ou mudança nas porinas bacterianas (Williams, 1999).

De acordo com Goel et al. (2011), A. baumannii é uma bactéria que tem obtido destaque no âmbito hospitalar, sendo que muitas vezes exibe um perfil de multirresistência aos antimicrobianos. Essa resistência observada em bactérias gramnegativas tornou-se um sério problema ao redor do globo, pois pode reduzir as opções de tratamento substancialmente. A resistência aos carbapenêmicos é um bom marcador para tais situações e é mais prevalente nos gêneros Acinetobacter, Pseudomonas e em bactérias da família Enterobacteriaceae (Theuretzbacher, 2017).

Apesar dos esforços de instituições que visam o uso racional de antimicrobianos e melhorias nos métodos de diagnósticos, o surgimento e disseminação desses patógenos gram-negativos resistentes a carbapenêmicos é um contribuinte significativo para a morbidade e mortalidade dos pacientes (CDC, 2009; WHO, 2015b). O principal motivo para isso é que existem poucos agentes antimicrobianos ativos para este grupo e o desenvolvimento de novas drogas parece uma realidade distante na conjectura atual (Laxminarayan et al., 2013; Patel \& Bonomo, 2013; Perez et al., 2007).

Visando entender a resistência microbiana para que pudesse otimizar a terapêutica para os agentes disponíveis atualmente, a OMS criou o GLASS (Global Antimicrobial Resistance Surveillance System), com o objetivo principal de embasar um plano global para o combate à resistência aos antimicrobianos e, por fim, fortalecer as evidências e colaborar com a tomada de decisões em escala nacional, regional e global (WHO, 2021). Para carbapenêmicos o sistema GLASS atualmente 
possui dados referentes a Acinetobacter spp., Klebsiella pneumoniae, Salmonella spp. e E. coli. advindos de países dos mais diversos tipos (WHO, 2020). Esses gêneros e espécies bacterianas fazem parte do grupo de desenvolvimento prioritário destacado pela OMS no Global priority list of antibiotic-resistant bacteria to guide research, discovery, and development of new antibiotics, quando apresentam o fenótipo de resistência aos carbapenêmicos (WHO, 2017).

É interessante destacar que já existiam programas mundiais para bactérias especificas como o Mycobacterium tuberculosis e a Neisseria gonorrhoeae. Também existindo programas regionais que monitoravam a resistência bacteriana de forma mais ampla, como o Central Asian and Eastern European Surveillance of Antimicrobial Resistance (CAESAR), European Antimicrobial Resistance Surveillance Network (EARS-Net) e a Latin American Antimicrobial Resistance Surveillance Network (ReLAVRA). Apesar desses programas serem exitosos, existiam ainda diversas lacunas como, por exemplo, a falta de padronização dos métodos de coleta de dados, compartilhamento e coordenação das iniciativas, fatores que dificultavam a produção de dados a nível global (WHO, 2015c). Esses dados para a resistência bacteriana eram relativamente limitados e fragmentados, apesar das evidências de que no resto do mundo os níveis de resistência estão aumentando (WHO, 2015a).

Falta de informações confiáveis é um dos obstáculos para a melhoria dos sistemas de saúde dos países em desenvolvimento. Um constante fluxo de dados é essencial para delimitar as necessidades sanitárias de uma população, orientando novos projetos e sua implementação (The Lancet, 2005). Países de baixa renda ou renda média baixa têm ainda menor taxa de adesão a sistemas de vigilância, representando um entrave para o desenvolvimento de um programa realmente global de monitoramento do consumo de antimicrobianos e resistência bacteriana (WHO, 2020).

Desse modo, esta pesquisa tem por objetivo discutir o nível de não-suscetibilidade da Acinetobacter spp. aos carbapenêmicos com dados encontrados no sistema GLASS, estratificados pelo nível de renda per capita dos países notificadores, destacando-se os países que possuem um grande nível de acometimento por este fenômeno, bem como aqueles que parecem controlar o mesmo, visando soluções.

\section{Metodologia}

Foi conduzido um estudo observacional retrospectivo sobre a não-susceptibilidade a carbapenêmicos da Acinetobacter spp., reportada ao sistema GLASS (WHO, 2021) da OMS referentes aos anos de 2016, 2017 e 2018. Esses dados foram obtidos através da revisão dos materiais suplementares eletrônicos dos relatórios GLASS Early Implementation Report: 20162017 (WHO, 2018), GLASS Early Implementation Report: 2017-2018 (WHO, 2019) e GLASS Early Implementation Report: 2020 (WHO, 2020), referentes aos anos de 2016, 2017 e 2018, respectivamente, e que no momento de elaboração deste artigo, fevereiro de 2021, representavam a totalidade dos dados disponíveis no sistema GLASS, no qual possuem certo atraso de sua publicação em vista da amplitude dos dados trabalhados.

A classificação da renda per capita e população total foram obtidos através do DataBank ${ }^{\circledR}$ do Banco Mundial, referentes aos anos de 2016, 2017 e 2018 (The World Bank, 2021). O banco mundial classifica o nível de renda per capita dos países em: renda baixa, renda média baixa, renda média alta e renda alta.

Os dados sobre a não-susceptibilidade a carbapenêmicos quando não reportados diretamente ao sistema GLASS eram estratificados através dos valores observados para o imipenem ou meropenem, sendo escolhido o que tivesse o maior número de testes de sensibilidade a carbapenêmicos. Em caso de empate, considerou-se o que possuía o maior valor de nãosuscetibilidade a fim de evitar duplicidade dos dados estudados. Essa adaptação foi feita baseada na recomendação inicial da implementação do GLASS (WHO, 2015b). Um isolado bacteriano é considerado não-susceptível a um agente antimicrobiano quando o resultado do seu antibiograma é resistente ou intermediário (CLSI, 2020).

Foi determinado o número de testes de sensibilidade a carbapenêmicos (TSC) por um milhão de habitantes (1M) reportados ao sistema GLASS, para cada um dos anos estudados, seguindo a equação: 


$$
\frac{\mathrm{TSC}}{1 \mathrm{M}}=\mathrm{N} N \text { de TSC realizados pelo país * } \frac{1000000}{\text { população do país no ano avaliado }}
$$

Posteriormente, foi calculada a média para as quatro faixas de renda consideradas pelo Banco Mundial, com determinação do erro padrão da média utilizando o intervalo de confiança de $95 \%(\alpha=5 \%)$ através do programa GraphPad Prism $8^{\circledast}$. O cálculo do intervalo de confiança teve por objetivo avaliar se existia diferença estatisticamente significante entre as faixas de renda per capita.

\section{Resultados e Discussão}

A falta de novos antimicrobianos exige um uso cauteloso dos agentes disponíveis. Quase todos os países enfrentam o aumento da resistência microbiana em geral, fato que não é verdadeiro somente para humanos, mas em boa parte da cadeia produtiva que depende deles (Harbarth et al., 2015).

A perda de eficácia contra patógenos comuns para os antimicrobianos não somente leva a um uso de medicamentos mais caros em países de renda alta, mas também contribui no aumento da mortalidade e morbidade em países baixa e média renda, onde nem sempre medicamentos alternativos estariam disponíveis, pois seu custo pode ser proibitivo dentro da realidade do país ou região em questão (Laxminarayan et al., 2013).

O gênero Acinetobacter spp. pode exibir mecanismos resistência a praticamente todas as classes de antimicrobianos existentes e possui uma grande capacidade de desenvolver novas formas de resistência. Seu sucesso em desencadear surtos deve-se a uma combinação de diversos fatores, dentre eles sua resistência ambiental e grande adaptabilidade. Geneticamente ela pode possuir os mesmos conjuntos de genes encontrados em outras espécies bacterianas como a Pseudomonas spp., Salmonella spp. e E. coli (Perez et al., 2007). Seu comportamento de resistência da A. baumannii é fortemente associado ao consumo de carbapenêmicos (Hsu et al., 2010; Sousa et al., 2013; Xu et al., 2013).

É interessante notar que conforme a faixa de renda per capita dos países aumenta, maior é a quantidade de participantes do sistema GLASS e a quantidade de TSCs/1M reportados ao sistema. No ano de 2018 as médias de TSCs/1M reportados ao sistema GLASS foram de 4,3 TSCs/1M, 16,1 TSCs/1M, 20,1 TSCs/1M, e os desvios padrões foram de $\pm 5,9$ $\mathrm{TSCs} / 1 \mathrm{M}, \pm 21,1 \mathrm{TSCs} / 1 \mathrm{M} \mathrm{e} \pm 20,8 \mathrm{TSC} / 1 \mathrm{M}$, em ordem crescente de faixa de renda (tabela 1). Infelizmente, a renda de um país influencia de forma considerável a sua capacidade de desenvolver sistemas de vigilância. Destaca-se que, apesar de 12 países de baixa renda estarem incluídos no ano 2018, nenhum reportou dados a respeito da resistência a carbapenêmicos da Acinetobacter spp. (WHO, 2020).

Existe a necessidade do fortalecimento da vigilância para a resistência bacteriana em países de baixa renda, e o envolvimento de países mais desenvolvidos pode ser essencial para que isso ocorra (LSHTM, 2016). O Fundo Fleming investe em alguns programas para acompanhamento da resistência bacteriana, todavia o início da maioria dos programas de apoio ocorreu após o ano de 2019, que até o momento desse estudo não possuía dados publicados (Fleming Fund, 2021). 
Tabela 1 - Adesão dos países em TSCs/1M durante os anos de 2016, 2017 e 2018 estratificados por renda per capita que reportaram testes de resistência a carbapenêmicos no sistema GLASS.

\begin{tabular}{|c|c|c|c|c|}
\hline & Baixa renda & Renda média baixa & Renda média alta & Alta renda \\
\hline \multicolumn{5}{|c|}{2016} \\
\hline Média & 2,1 & 7,0 & 13,6 & 10,7 \\
\hline Desvio padrão & 2,9 & 6,3 & 19,7 & 8,3 \\
\hline Quantidade de países aderentes & 2 & 3 & 5 & 9 \\
\hline \multicolumn{5}{|l|}{ 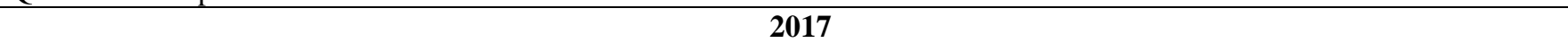 } \\
\hline Média & 3,5 & 3,7 & 13,1 & 17,5 \\
\hline Desvio padrão & 5,7 & 7,2 & 21,1 & 21,5 \\
\hline Quantidade de países aderentes & 3 & 9 & 11 & 24 \\
\hline \multicolumn{5}{|l|}{ 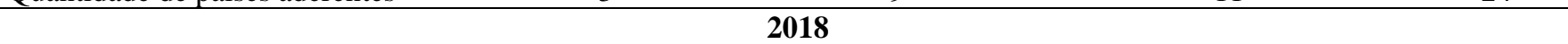 } \\
\hline Média & - & 4,3 & 16,1 & 20,1 \\
\hline Desvio padrão & - & 5,9 & 21,1 & 20,8 \\
\hline Quantidade de países aderentes & - & 9 & 15 & 25 \\
\hline
\end{tabular}

Fonte: Dados da pesquisa (2021).

O alto valor dos desvios padrão frente a média revela que existe uma grande variância entre os países de cada grupo. Fator observável no Gráfico 1, o qual mostra que países de diferentes faixas de renda muitas vezes possuem valores próximos de TSCs/1M. Utilizando um intervalo de confiança de 95\%, observa-se que não existe diferença estatisticamente significante entre os diferentes níveis de renda per capita e a quantidade de TSCs/1M. Essa conclusão é possível por em nenhum momento os intervalos de confiança em vermelho no Gráfico 1 deixaram de tocar o eixo y (Patino \& Ferreira, 2015).

Gráfico 1. Adesão dos países ao GLASS em TSCs/1M durante os anos de 2016, 2017 e 2018, estratificados por renda per capita.

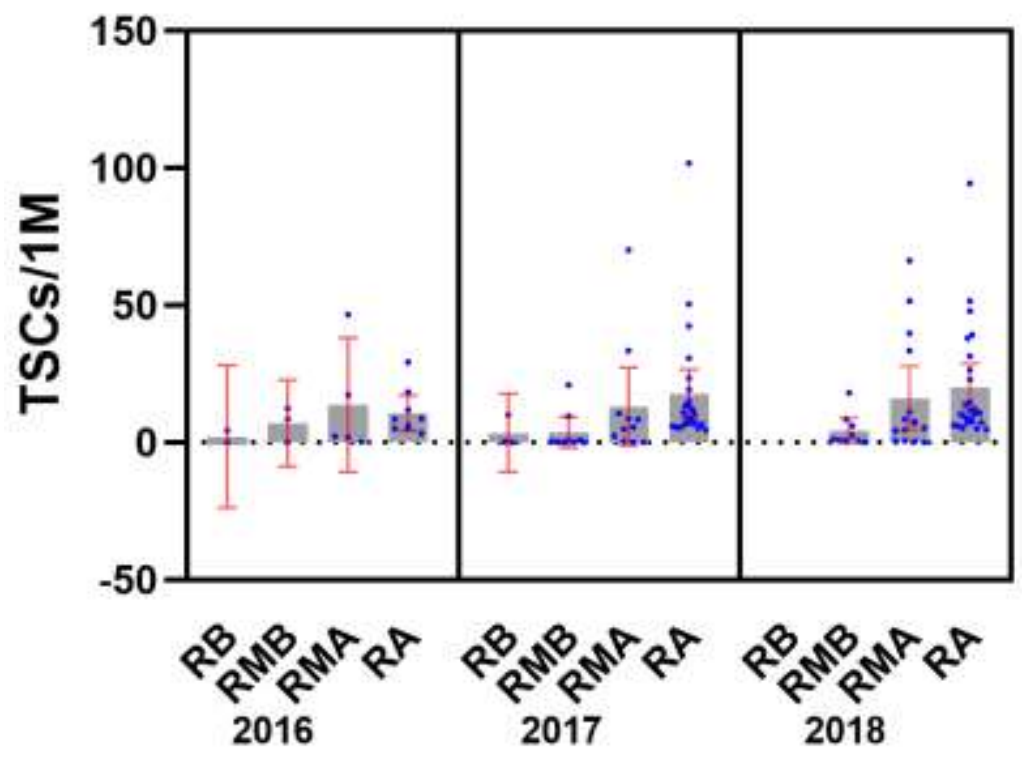

Legenda: RB: Renda baixa, RMB; Renda média baixa; Renda média alta; Renda alta; Barras cinzas: Porcentagem da média de não-suscetíveis para cada nível de renda por ano; Barras vermelhas: Erro padrão da média para cada nível de renda por ano, utilizando o intervalo de confiança de $95 \%(\alpha=5 \%)$; Pontos azuis porcentagem dos não-suscetíveis para cada nível de renda por ano. Fonte: Dados da pesquisa (2021).

Nota-se uma concentração de países de alta renda com baixa suscetibilidade a carbapenêmicos, valores abaixo de 10\%. Eles são França, Países Baixos, Áustria, Alemanha, Suíça, Suécia, Reino Unido da Grã-Bretanha e Irlanda do Norte, Irlanda, Japão, Finlândia, Malta e Noruega. Para nenhum dos outros grupos foi possível avaliar esse comportamento (Gráfico 2). Existe uma tendência na menor resistência a carbapenêmicos para a Acinetobacter spp., conforme o IDH aumenta, já que os 
dois países de renda alta per capita que possuem índices de resistência acima de 90\% (Grécia e Croácia) têm seu IDH abaixo da $32^{\mathrm{a}}$ posição no rank de 2020. Por outro lado, aqueles com menores índices de resistência estão em sua maioria acima da $20^{\mathrm{a}}$ posição, com exceção de Malta e a França (UNDP, 2021). Esses países podem aproveitar sua posição privilegiada na assistência dos piores casos observados, pois a cooperação entre eles é essencial para expansão dos programas de controle de antimicrobianos (United Nations, 2019).

Os níveis gerais de não-suscetibilidade aos carbapenêmicos da Acinetobacter spp. foram elevados. Nota-se que somente os países de renda alta têm o valor médio abaixo de 50\%. Contudo, mesmo em países de alta renda, para o ano de 2018, existem aqueles em que a não-suscetibilidade ultrapassa os $90 \%$, Valor observado para a Grécia $(92,8 \%$ - 94,38 TSCs/1M) e a Croácia (96,8\% - 37,9 TSCs/1M) (Grafico 1). Para a Grécia, esse valor é condizente com um estudo de representatividade nacional que encontrou a prevalência de A. baumannii resistente a carbapenêmicos de 83\% (Kritsotakis et al., 2017). Em outro estudo que avaliou a resistência da Acinetobacter spp. a carbapenêmicos no continente europeu, utilizando dados da EARS-Net, mostrou-se que a região da Europa meridional é aquela que possui a maior resistência dentre as regiões do continente. Essa região é onde se localizam a Croácia e a Grécia. O valor da resistência para ano de 2017, último ano avaliado pelo estudo, ficou acima dos 75\% (Ayobami et al., 2020).

Os valores de TSCs/1M não demostram que possa ocorrer uma distorção por notificação de ambientes de alta resistência, local onde se poderia esperar esse fenômeno. Para os dois países de alta renda, a abrangência de seu plano de vigilância é nacional (WHO, 2020). E sua média de TSCs/1M é acima da média de todos os grupos, demonstrando boa representatividade na amostra estudada.

Dentre os países de renda média alta com altos níveis de resistência, destacam-se a Federação Russa (90,8\% - 0,5 TSCs/1M) e a Bósnia e Herzegovina (93,2\% - 39,7 TSCs/1M) (Gráfico 2). Apesar de não terem sido encontrados artigos de alcance nacional para estes dois países, já existem relatos de A. baumannii produtoras de enzimas de clivagem de espectro ampliado para estes países (Mayanskiy et al., 2017; Petrović et al., 2018). Quanto à quantidade de TSCs/1M, a Federação Rússia encontra-se bem abaixo dos valores de seu grupo - apesar de seu plano de vigilância ter amplitude nacional - o que em si pode gerar dados duvidosos. Apesar disso, a Bósnia e Herzegovina apresenta uma confiabilidade consideravelmente maior quanto à quantidade de TSCs/1M, entretanto seus dados advêm de somente uma instituição hospitalar, o que em si torna-se uma distorção do possível quadro nacional (WHO, 2020).

Dentre os países de renda média baixa, os destaques foram o Egito (76,5\% - 1,8 TSCs/1M) e a Tunísia $(83,3 \%$ - 18,15 TSCs/1M (Gráfico 2). Nesses dois países também foi possível observar artigos que demonstram a presença de A. baumannii produtoras de enzimas de espectro ampliado (Amiri et al., 2017; Jaidane et al., 2018; Mansour, 2018). Ambos os países não possuem planos de vigilância com cobertura nacional, sendo isso um possível ponto de distorção (WHO, 2020). Não obstante, a Tunísia apresenta uma cobertura de TSCs/1M bem acima do seu grupo, com mais de 2 desvios padrões de diferença da média dele, fato que a destaca em comparação ao seu grupo.

Os países de renda média alta foram aqueles que apresentaram o maior nível de não-suscetibilidade no ano de 2018 (Gráfico 2). Entre os anos de 2000 e 2010, o consumo de antimicrobianos aumentou em 35\%. Países como o Brasil, Federação Russa, Índia, China e África do Sul foram responsáveis por 76\% desse aumento. Para os carbapenêmicos esse aumento foi de 45\% (Van Boeckel et al., 2014). Esse aumento do consumo nesses países pode explicar a maior não-suscetibilidade observada, já que todos são países de renda média alta, com exceção da Índia (The World Bank, 2021). Contudo, este país sofre de dois problemas graves: um grande consumo de antimicrobianos em comparação a outros países (Van Boeckel et al., 2014) e um uso extensivo de antimicrobianos sem prescrição (McGettigan et al., 2019). 
Gráfico 2. Porcentagem dos isolados de Acinetobacter spp. não-suscetíveis a carbapenêmicos reportados ao sistema GLASS.

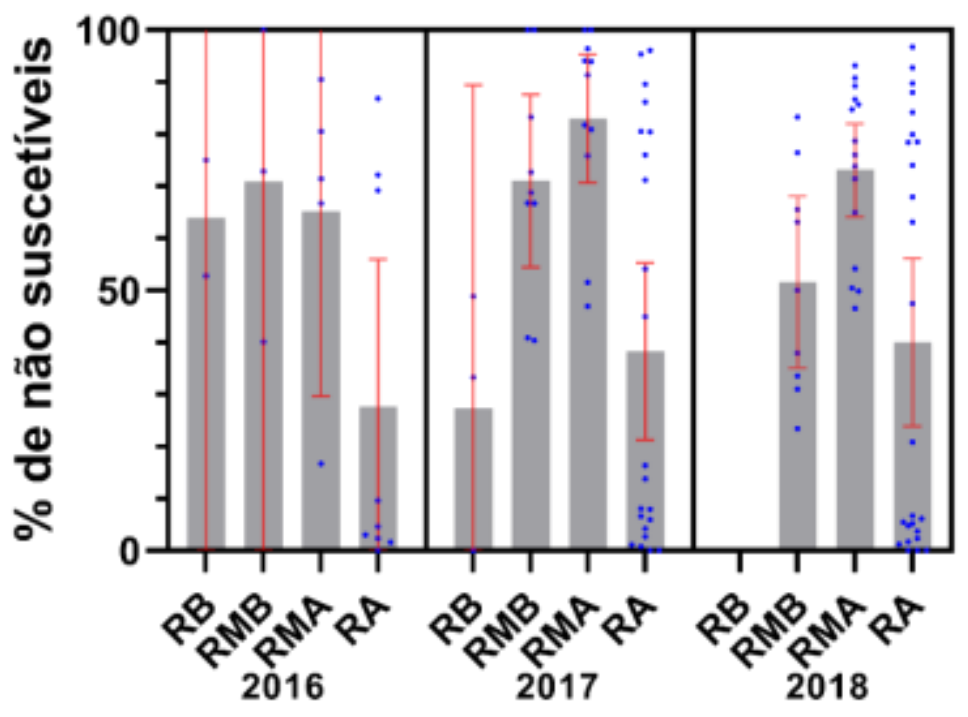

Legenda: RB: Renda baixa, RMB; Renda média baixa; Renda média alta; Renda alta; Barras cinzas: Porcentagem da média de não-suscetíveis para cada nível de renda por ano; Barras vermelhas: Erro padrão da média para cada nível de renda por ano, utilizando o intervalo de confiança de $95 \%(\alpha=5 \%)$; Pontos azuis porcentagem dos não-suscetíveis para cada nível de renda por ano. Fonte: Dados da pesquisa (2021).

O Fundo Fleming, de origem britânica, destaca que os países mais ricos devem contribuir no enfrentamento da resistência bacteriana (Fleming Fund, 2021). Faz-se necessária uma união global a fim de buscar uma solução plena para esse problema que atinge a todos (United Nations, 2019).

\section{Conclusão}

Os dados trabalhados nesse artigo mostram um nível preocupante de resistência a carbapenêmicos observados no sistema GLASS. Dentro mesmo dos países ricos há aqueles que possuem níveis alarmantes de não-susceptibilidade. Para os países mais pobres destaca-se uma escassez de dados mais completos. Existe uma necessidade de expansão dos dados coletados, visando uma melhor representatividade deles frente às diferentes realidades de países tão distintos entre si.

Como o prosseguimento do GLASS, será possível construir um modelo estatístico com maior poder analítico, uma vez que, infelizmente, 3 pontos de observação em si podem ser um fator limitante nesse tipo de análise. Destaca-se que com a adição de mais 4 pontos observação poderemos construir uma curva de tendência com maior capacidade preditiva.

Ressalta-se ainda que o GLASS é um programa em constante evolução e, com a adição recente do módulo de avaliação do consumo de antimicrobianos, seremos capazes de estudar a relação entre o consumo e a resistência bacteriana com um modelo robusto e de escala mundial.

\section{Agradecimentos}

Este estudo foi realizado com o apoio da CAPES (Coordenação de Aperfeiçoamento de Pessoal de Nível Superior) e do PPGCF - UFPI (Programa de Pós-graduação em Ciências Farmacêuticas - Universidade Federal do Piauí).

\section{Referências}

Amiri, S., Hammami, S., Amoura, K., Dekhil, M., \& Boubaker, I. B.-B. (2017). Characterization of carbapenem resistant Acinetobacter baumannii isolated from intensive care units in two teaching hospitals from Algeria and Tunisia. Pan African Medical Journal, 28. https://doi.org/10.11604/pamj.2017.28.19.9713 
Ayobami, O., Willrich, N., Suwono, B., Eckmanns, T., \& Markwart, R. (2020). The epidemiology of carbapenem-non-susceptible Acinetobacter species in Europe: analysis of EARS-Net data from 2013 to 2017. Antimicrobial Resistance \& Infection Control, 9(1), 89. https://doi.org/10.1186/s13756-020-00750-5

CDC. (2009). Guidance for control of infections with carbapenem-resistant or carbapenemase-producing Enterobacteriaceae in acute care facilities. $M M W R$. Morbidity and Mortality Weekly Report, 58(10), 256-260.

CLSI. (2020). CLSI AST News Update. CLSI. https://clsi.org/media/3486/clsi_astnewsupdate_january2020.pdf

Fleming Fund. (2021). Aims \& Values. 2021. https://www.flemingfund.org/about-us/our-aims/

Goel, N., Wattal, C., Oberoi, J. K., Raveendran, R., Datta, S., \& Prasad, K. J. (2011). Trend analysis of antimicrobial consumption and development of resistance in non-fermenters in a tertiary care hospital in Delhi, India. Journal of Antimicrobial Chemotherapy, 66(7), 1625-1630. https://doi.org/10.1093/jac/dkr167

Harbarth, S., Balkhy, H. H., Goossens, H., Jarlier, V., Kluytmans, J., Laxminarayan, R., Saam, M., Van Belkum, A., \& Pittet, D. (2015). Antimicrobial resistance: one world, one fight! Antimicrobial Resistance and Infection Control, 4(1), 49. https://doi.org/10.1186/s13756-015-0091-2

Hawkey, P. M., \& Livermore, D. M. (2012). Carbapenem antibiotics for serious infections. BMJ, 344(may31 1), e3236-e3236. https://doi.org/10.1136/bmj.e3236

Hsu, L.-Y., Tan, T.-Y., Tam, V. H., Kwa, A., Fisher, D. A., \& Koh, T.-H. (2010). Surveillance and Correlation of Antibiotic Prescription and Resistance of Gram-Negative Bacteria in Singaporean Hospitals. Antimicrobial Agents and Chemotherapy, 54(3), 1173-1178. https://doi.org/10.1128/AAC.01076-09

Jaidane, N., Naas, T., Oueslati, S., Bernabeu, S., Boujaafar, N., Bouallegue, O., \& Bonnin, R. A. (2018). Whole-genome sequencing of NDM-1-producing ST85 Acinetobacter baumannii isolates from Tunisia. International Journal of Antimicrobial Agents, 52(6), 916-921. https://doi.org/10.1016/j.ijantimicag.2018.05.017

Kritsotakis, E. I., Kontopidou, F., Astrinaki, E., Roumbelaki, M., Ioannidou, E., \& Gikas, A. (2017). Prevalence, incidence burden, and clinical impact of healthcare-associated infections and antimicrobial resistance: a national prevalent cohort study in acute care hospitals in Greece. Infection and Drug Resistance, 10, 317-328. https://doi.org/10.2147/IDR.S147459

Laxminarayan, R., Duse, A., Wattal, C., Zaidi, A. K. M., Wertheim, H. F. L., Sumpradit, N., Vlieghe, E., Hara, G. L., Gould, I. M., Goossens, H., Greko, C., So, A. D., Bigdeli, M., Tomson, G., Woodhouse, W., Ombaka, E., Peralta, A. Q., Qamar, F. N., Mir, F., ... Cars, O. (2013). Antibiotic resistance- -the need for global solutions. The Lancet Infectious Diseases, 13(12), 1057-1098. https://doi.org/10.1016/S1473-3099(13)70318-9

LSHTM. (2016). AMR Surveillance in low- and middle-income settings. London School of Hygiene \& Tropical Medicine. https://wellcomeopenresearch.s3.amazonaws.com/supplementary/12527/99f63366-743d-473c-b3ad-e96403e4ab3e.pdf

Mansour, W. (2018). Tunisian antibiotic resistance problems: three contexts but one health. African Health Sciences, 18(4), 1202. https://doi.org/10.4314/ahs.v18i4.41

Mayanskiy, N., Chebotar, I., Alyabieva, N., Kryzhanovskaya, O., Savinova, T., Turenok, A., Bocharova, Y., Lazareva, A., Polikarpova, S., \& Karaseva, O. (2017). Emergence of the Uncommon Clone ST944/ST78 Carrying bla OXA-40-like and bla CTX-M-like Genes Among Carbapenem-Nonsusceptible Acinetobacter baumannii in Moscow, Russia. Microbial Drug Resistance, 23(7), 864-870. https://doi.org/10.1089/mdr.2016.0302

McGettigan, P., Roderick, P., Kadam, A., \& Pollock, A. (2019). Threats to global antimicrobial resistance control: Centrally approved and unapproved antibiotic formulations sold in India. British Journal of Clinical Pharmacology, 85(1), 59-70. https://doi.org/10.1111/bcp.13503

Patel, G., \& Bonomo, R. A. (2013). "Stormy waters ahead": global emergence of carbapenemases. Frontiers in Microbiology, 4, 48. https://doi.org/10.3389/fmicb.2013.00048

Patino, C. M., \& Ferreira, J. C. (2015). Confidence intervals: a useful statistical tool to estimate effect sizes in the real world. Jornal Brasileiro de Pneumologia, 41(6), 565-566. https://doi.org/10.1590/s1806-37562015000000314

Perez, F., Hujer, A. M., Hujer, K. M., Decker, B. K., Rather, P. N., \& Bonomo, R. A. (2007). Global Challenge of Multidrug-Resistant Acinetobacter baumannii. Antimicrobial Agents and Chemotherapy, 51(10), 3471-3484. https://doi.org/10.1128/AAC.01464-06

Petrović, T., Uzunović, S., Barišić, I., Luxner, J., Grisold, A., Zarfel, G., Ibrahimagić, A., Jakovac, S., Slaćanac, D., \& Bedenić, B. (2018). Arrival of carbapenem-hydrolyzing-oxacillinases in Acinetobacter baumannii in Bosnia and Herzegovina. Infection, Genetics and Evolution, 58, 192-198. https://doi.org/10.1016/j.meegid.2017.12.021

Sousa, D., Castelo-Corral, L., Gutierrez-Urbon, J.-M., Molina, F., Lopez-Calvino, B., Bou, G., \& Llinares, P. (2013). Impact of ertapenem use on Pseudomonas aeruginosa and Acinetobacter baumannii imipenem susceptibility rates: collateral damage or positive effect on hospital ecology? Journal of Antimicrobial Chemotherapy, 68(8), 1917-1925. https://doi.org/10.1093/jac/dkt091

The Lancet. (2005). Stumbling around in the dark. Lancet (London, England), 365(9476), 1983. https://doi.org/10.1016/S0140-6736(05)66671-8

The World Bank. (2021). DataBank. The World Bank. https://databank.worldbank.org/home.aspx

Theuretzbacher, U. (2017). Global antimicrobial resistance in Gram-negative pathogens and clinical need. Current Opinion in Microbiology, 39, 106-112. https://doi.org/10.1016/j.mib.2017.10.028

UNDP. (2021). Human Development Report 2020. United Nations Development Programme (UNDP). http://hdr.undp.org/sites/default/files/hdr2020.pdf

United Nations. (2019). Follow-up to the political declaration of the high-level meeting of the General Assembly on antimicrobial resistance. United Nations. https://undocs.org/en/A/73/869 
Van Boeckel, T. P., Gandra, S., Ashok, A., Caudron, Q., Grenfell, B. T., Levin, S. A., \& Laxminarayan, R. (2014). Global antibiotic consumption 2000 to 2010: an analysis of national pharmaceutical sales data. The Lancet Infectious Diseases, 14(8), 742-750. https://doi.org/10.1016/S1473-3099(14)70780-7

WHO. (2015a). Global Action Plan: Antimicrobial Resistance. https://apps.who.int/iris/bitstream/handle/10665/193736/9789241509763_eng.pdf?sequence=1

WHO. (2015b). Global Antimicrobial Resistance Surveillance System: Manual for Early Implementation. https://apps.who.int/iris/bitstream/handle/10665/188783/9789241549400_eng.pdf?sequence=1

WHO. (2015c). Global Antimicrobial Resistance Surveillance System: Manual for Early Implementation. WHO. https://apps.who.int/iris/bitstream/handle/10665/188783/9789241549400_eng.pdf?sequence=1

WHO. (2017). Global priority list of antibiotic-resistant bacteria to guide research, discovery, and development of new antibiotics. World Health Organization (WHO). https://www.who.int/medicines/publications/WHO-PPL-Short_Summary_25Feb-ET_NM_WHO.pdf

WHO. (2018). GLASS Early Implementation Report: 2016-2017. WHO. https://www.who.int/glass/resources/publications/early-implementation-report/en/

WHO. (2019). GLASS Early Implementation Report: 2017-2018. WHO. https://www.who.int/glass/resources/publications/early-implementation-report-20172018/en/

WHO. (2020). GLASS Early Implementation Report: 2020. WHO. https://www.who.int/glass/resources/publications/early-implementation-report-2020/en/

WHO. (2021). Global Antimicrobial Resistance Surveillance System (GLASS). WHO. https://www.who.int/glass/en/

Williams, J. D. (1999). Beta-lactamases and beta-lactamase inhibitors. International Journal of Antimicrobial Agents, 12 Suppl 1, S3-7; discussion S26-7. https://doi.org/10.1016/s0924-8579(99)00085-0

Xu, J., Sun, Z., Li, Y., \& Zhou, Q. (2013). Surveillance and Correlation of Antibiotic Consumption and Resistance of Acinetobacter baumannii complex in a Tertiary Care Hospital in Northeast China, 2003-2011. International Journal of Environmental Research and Public Health, 10(4), 1462-1473. https://doi.org/10.3390/ijerph10041462 